\title{
Evaluation of a new and automated electrochemiluminescence immunoassay for plasma sFIt-1 and PIGF levels in women with preeclampsia
}

\author{
Akihide Ohkuchi, Chikako Hirashima, Hirotada Suzuki, Kayo Takahashi, Mika Yoshida, Shigeki Matsubara, \\ and Mitsuaki Suzuki
}

The first commercial automated immunoassays specific for soluble fms-like tyrosine kinase 1 (sFlt-1) and placental growth factor (PIGF) (Elecsys sFlt-1 and Elecsys PIGF, respectively) have recently been introduced. We constructed reference range values of plasma levels of sFIt-1 and PIGF, and the sFIt-1/PIGF ratio using Elecsys sFIt-1 and Elecsys PIGF during the second half of pregnancy and evaluated their sensitivity and specificity for the diagnosis of preeclampsia. Plasma samples were collected from 144 normal pregnant women at 19-25, 27-31 and 34-38 weeks of gestation and from 34 women with preeclampsia. The most appropriate reference range curves for plasma levels of sFIt-1 and PIGF, and the sFIt-1/PIGF ratio are presented as quadratic curves after logarithmic transformation. The sFIt-1/PIGF ratio showed the best diagnostic power for both early-onset and late-onset preeclampsia. In addition, a cutoff value of $\mathbf{4 5}$ for the sFIt-1/PIGF ratio resulted in the best sensitivity and specificity for the diagnosis of all preeclampsia (97 and $95 \%$, respectively), and for the diagnosis of early-onset preeclampsia (100 and $95 \%$, respectively). Using another 50 pairs of serum and plasma samples, including those from normal pregnant women and preeclamptic women, the plasma recovery rates of sFIt-1 and PIGF were 0.89 and 0.85 , respectively; the correlation determinations between serum and plasma samples were 0.999 for sFIt-1, 0.990 for PIGF and 0.987 for sFIt-1/PIGF ratio. In conclusion, measurement of the plasma sFIt-1/PIGF ratio determined by Elecsys sFIt-1 and Elecsys PIGF and using a cutoff value of $\mathbf{4 5}$ might assist in the diagnosis of preeclampsia, especially for early-onset preeclampsia. Hypertension Research (2010) 33, 422-427; doi:10.1038/hr.2010.15; published online 12 February 2010

Keywords: diagnosis; PIGF; preeclampsia, sFIt-1; sFIt-1/PIGF ratio

\section{INTRODUCTION}

Immunoassays for soluble fms-like tyrosine kinase 1 (sFlt-1) and placental growth factor (PlGF) (Elecsys sFlt-1 and Elecsys PlGF, respectively; Roche Diagnostics, Penzberg, Germany) have been recently developed for in vitro diagnostic testing of preeclampsia (PE). We have previously examined the levels of sFlt-1 and PlGF, and the sFlt-1/PlGF ratio in women with PE using the enzyme-linked immunosorbent assay (ELISA) kits developed by R\&D Systems (Minneapolis, MN, USA). ${ }^{1,2}$ However, ELISA kits have been restricted for research use only, so we could not use these values in diagnostic procedures for PE. Elecsys sFlt-1 and Elecsys PlGF measurements are fully automated and are performed using commercially available laboratory analytical instruments for electrochemiluminescence immunoassay systems, such as the Roche Modular E170 analyzer, which has been introduced into many laboratories. In addition, sFlt-1 and PlGF levels can be obtained in only $18 \mathrm{~min}$. Because these kits were developed for clinical use, it is very important for clinicians to be aware of their clinical sensitivity and specificity for the diagnosis of PE.
Our aim was to establish a reference range for plasma levels of sFlt-1 and PlGF, and the sFlt-1/PlGF ratio using Elecsys sFlt-1 and Elecsys PlGF during the second half of pregnancy and to evaluate their sensitivity and specificity for the diagnosis of PE.

\section{METHODS}

Subjects and procedures

We obtained written informed consent from all women, as well as approval from the ethics committee of our institute. Although we had a contract with Roche Diagnostics to use Elecsys sFlt-1 and Elecsys PIGF for this study, there are no conflicts of interest in regards to this paper. The study subjects consisted of two groups: one to establish a reference range for plasma sFlt-1 and PlGF levels and the sFlt-1/PlGF ratio according to gestational age, and the other to evaluate those indices after the clinical manifestation of early- or late-onset PE. The study subjects were all Japanese women with singleton pregnancies. The first group consisted of 187 pregnant women who had blood samples taken two or three times at 19-25, 27-31 and 34-38 weeks of gestation; the samples were collected serially between January 2006 and April 2007. After excluding women with pregnancy-induced hypertension, small-for-gestational-age infants or 
preterm delivery; those with a history of hypertension and those who delivered in another clinic/hospital, the remaining 144 pregnant women, from whom 338 samples were collected, were used to establish reference range values for plasma sFlt- 1 and PlGF levels and the sFlt-1/PlGF ratio using Elecsys sFlt- 1 and Elecsys PIGF. The second group consisted of 34 referred women with PE who had blood samples collected as soon as possible after admission to our hospital due to the clinical manifestation of PE between April 2004 and April 2007.

In addition, we later collected another 50 serum and plasma samples to evaluate recovery rates and determine the sFlt- 1 and PlGF correlation using these new analysis kits. We also randomly collected 39 plasma samples from 21 women without PE and 18 women with PE to evaluate plasma levels of sFlt-1 and PIGF measured by ELISA kits developed by R\&D Systems (Human Soluble VEGF R1/Flt-1 Immunoassay, DVR100B; Human PlGF Immunoassay, DPG00) using the same samples used in this study.

Blood samples were collected in tubes containing ethylenediaminetetraacetic acid. Blood samples were centrifuged at $4^{\circ} \mathrm{C}$ at 2500 r.p.m. for $15 \mathrm{~min}$. Samples were stored at $-20^{\circ} \mathrm{C}$ until use. The sFlt- 1 and PlGF electrochemiluminescence immunoassays using Elecsys sFlt-1 and Elecsys PlGF were performed in duplicate using Modular Analytics E 170 (Roche Diagnostics) according to the manufacturer's instructions. The minimal detectable doses in the assays for serum sFlt- 1 and PlGF were 15 and $10 \mathrm{pg} / \mathrm{ml}$, respectively; the intra-assay and inter-assay coefficients of variation for serum sFlt- 1 were $0.5-6.8$ and $0.7-11 \%$, respectively; the intra-assay and inter-assay coefficients of variation for serum PlGF were $0.6-2.6$ and $0.6-5.9 \%$, respectively (according to the manufacturer's information).

\section{Definitions of PE and small-for-gestational-age infants}

We defined PE according to the definition and classification of pregnancyinduced hypertension (2004) of the Japan Society for the Study of Hypertension in Pregnancy. ${ }^{3}$ Briefly, PE was defined as hypertension with proteinuria that occurred after 20 weeks of gestation. Superimposed PE was defined as chronic hypertension diagnosed before pregnancy or before 20 weeks of gestation, followed by the occurrence of proteinuria. In this study, superimposed PE was included in the category of PE. Proteinuria was defined as equal to or more than $300 \mathrm{mg}$ of protein per day from 24 -h urine collection. If only test tape was available, repeated semiquantitative test results of $1+$, which represents $30 \mathrm{mg}$ per $100 \mathrm{ml}$ of protein or more, were considered as a positive result. We defined $\mathrm{PE}$ in subjects whose systolic blood pressure was $140-159 \mathrm{~mm} \mathrm{Hg}$ and/or whose diastolic blood pressure was $90-109 \mathrm{~mm} \mathrm{Hg}$ as mild $\mathrm{PE}$, and in subjects whose systolic blood pressure was equal to or greater than $160 \mathrm{~mm} \mathrm{Hg}$ and/or diastolic blood pressure was equal to or greater than $110 \mathrm{~mm} \mathrm{Hg}$ as severe PE. We defined $\mathrm{PE}$ with onset earlier than 32 weeks of gestation as early-onset (EO) PE, and onset at 32 weeks of gestation or later as late-onset (LO) PE. We diagnosed women with the following three findings-serum lactate dehydrogenase levels more than 1.5-fold the upper limit, serum aspartate aminotransferase levels 1.5fold more than the upper limit in our institute and low platelet counts $\left(<10 \times 10^{4}\right.$ per $\left.\mu \mathrm{l}\right)$ - as having hemolysis, elevated liver enzymes and low platelet syndrome. We defined infants as small-for-gestational-age when they had a birth weight below the 10th percentile for the Japanese population. ${ }^{4}$

\section{Recovery rates of plasma samples and correlation determinations between serum and plasma samples for Elecsys sFlt-1 and Elecsys PIGF}

The plasma recovery rates of sFlt- 1 and PlGF were 0.89 and 0.85 , respectively. The correlation determinations between serum and plasma samples were 0.999 for sFlt-1, 0.990 for PlGF and 0.987 for the sFlt-1/PlGF ratio.

\section{Correlation determinations between ELISA (R\&D) and Elecsys (Roche Diagnostics) for plasma sFlt-1 and PIGF levels and the sFlt-1/PIGF ratio}

Using only samples from women with $\mathrm{PE}$, the correlation determinations between ELISA and Elecsys for plasma sFlt-1 and PlGF levels and the sFlt-1/ PlGF ratio were $0.983,0.764$ and 0.988 , respectively. Using only samples from women without PE, the correlation determinations between ELISA and Elecsys for plasma sFlt-1 and PlGF levels and the sFlt-1/PlGF ratio were 0.956, 0.901 and 0.994 , respectively. Using all samples, the correlation determinations between ELISA and Elecsys for plasma sFlt-1 and PIGF levels and sFlt-1/PlGF ratio were $0.989,0.923$ and 0.989 , respectively.

\section{Statistical analysis}

We calculated the standard deviation scores (s.d.s.) of $\log _{10} \mathrm{SFlt}-1, \log _{10} \mathrm{PlGF}$ and $\log _{10}($ sFlt-1/PlGF) as $(A-B) / C$ (where $A$ is the raw value, $B$ the mean and $C$ the s.d.). The results are presented as the mean \pm s.d. For sFlt-1, PlGF and the sFlt-1/ PlGF ratio; statistical testing was conducted after logarithmic transformation because it was shown that logarithmic values in pregnant controls closely resembled normal distributions using normal proportion-proportion (P-P) plots and the Kolmogonov-Smirnov test. The unpaired $t$-test was used to compare two continuous variables if appropriate. The $\chi^{2}$-test or Fisher's exact test was used to compare categorical data. Fitted curve estimation was performed using built-in software within the SPSS software package (version 13.0J for Windows, Chicago, IL, USA). A value of $P<0.05$ was considered as significant.

\section{RESULTS}

Among the 144 normal pregnant women used to construct gestational-age-specific normal reference values, $48 \%$ of the women were nulliparous. The mean gestational age at delivery was $39.5 \pm 1.4$ weeks of gestation, and the mean birth weight was $3086 \pm 352 \mathrm{~g}$. Thirty-seven percent of the newborns were delivered transabdominally.

Using normal P-P plots, we checked the distributions of sFlt-1, PlGF and the sFlt-1/PlGF ratio at 20,21, 22, 23, 28, 29 and 37 weeks of gestation, when at least 10 samples were collected each week, and found that the values after logarithmic transformation showed a close to normal distribution. The mean $\log _{10}$ sFlt-1 during the second half of pregnancy could be represented by a quadratic curve $\left(y=0.00215 x^{2}-\right.$ $0.105 x+4.341$, where $y$ is mean $\log _{10}$ sFlt- 1 and $x$ weeks of gestation) (Figure 1a). Levene statistics of $\log _{10}$ sFlt-1 during the seven time periods were not significant $(P=0.297)$, so we hypothesized that the s.d. of $\log _{10}$ sFlt-1 each week during the second half of pregnancy was constant. We tried to find the best-fit constant value of the s.d. and found that the s.d. each week could be represented by a constant value of 0.207 . Likewise, the mean $\log _{10} \mathrm{PlGF}$ during the second half of pregnancy could be represented by a quadratic curve $\left(y=-0.00453 x^{2}+0.259 x-0.914\right)$ (Figure 1b). The results of the Levene statistics of $\log _{10} \mathrm{PlGF}$ during the seven time periods were significant $(P=0.002)$, and the s.d. tended to increase according to the gestational week, so the best-fit function by which the s.d. each week could be represented was linear $(y=0.0116 x+0.060)$. The distribution of $\log _{10}$ (sFlt-1/PlGF) during the second half of pregnancy could also be represented by a quadratic curve $\left(y=0.00668 x^{2}-0.382 x+5.265\right)$ (Figure 1c). Levene statistics of $\log _{10}(\mathrm{sFlt}-1 / \mathrm{PlGF})$ during the seven time periods were significant $(P<0.001)$, and the s.d. tended to increase according to gestational age, so the best-fit function by which the s.d. each week could be represented was linear $(y=0.012 x-0.006)$.

Women with EO-PE had small-for-gestational-age infants more frequently than those with LO-PE (Table 1). The level of maximum systolic blood pressure in women with EO-PE was higher than that in women with LO-PE.

Both EO-PE and LO-PE affected the plasma sFlt-1 and PlGF levels and the sFlt-1/PlGF ratio. Women with EO-PE had lower levels of $\log _{10} \mathrm{PlGF}$ and higher levels of $\log _{10}(\mathrm{sFlt}-1 / \mathrm{PlGF})$ than those with LO-PE (Table 2). Moreover, sFlt-1, PlGF and the sFlt-1/PlGF ratio deviated more from the normal reference curve in women with EO-PE than in those with LO-PE (Figure 1). These findings were supported statistically by the almost 1.5 - to 2.5 -fold difference in the average $\log _{10}$ sFlt-1 s.d.s., $\log _{10}$ PlGF s.d.s. and $\log _{10}($ sFlt-1/PlGF) s.d.s. between the two groups (Table 2). These results suggest that the increased sFlt-1 level, decreased PlGF level and increased sFlt-1/PlGF ratio may 

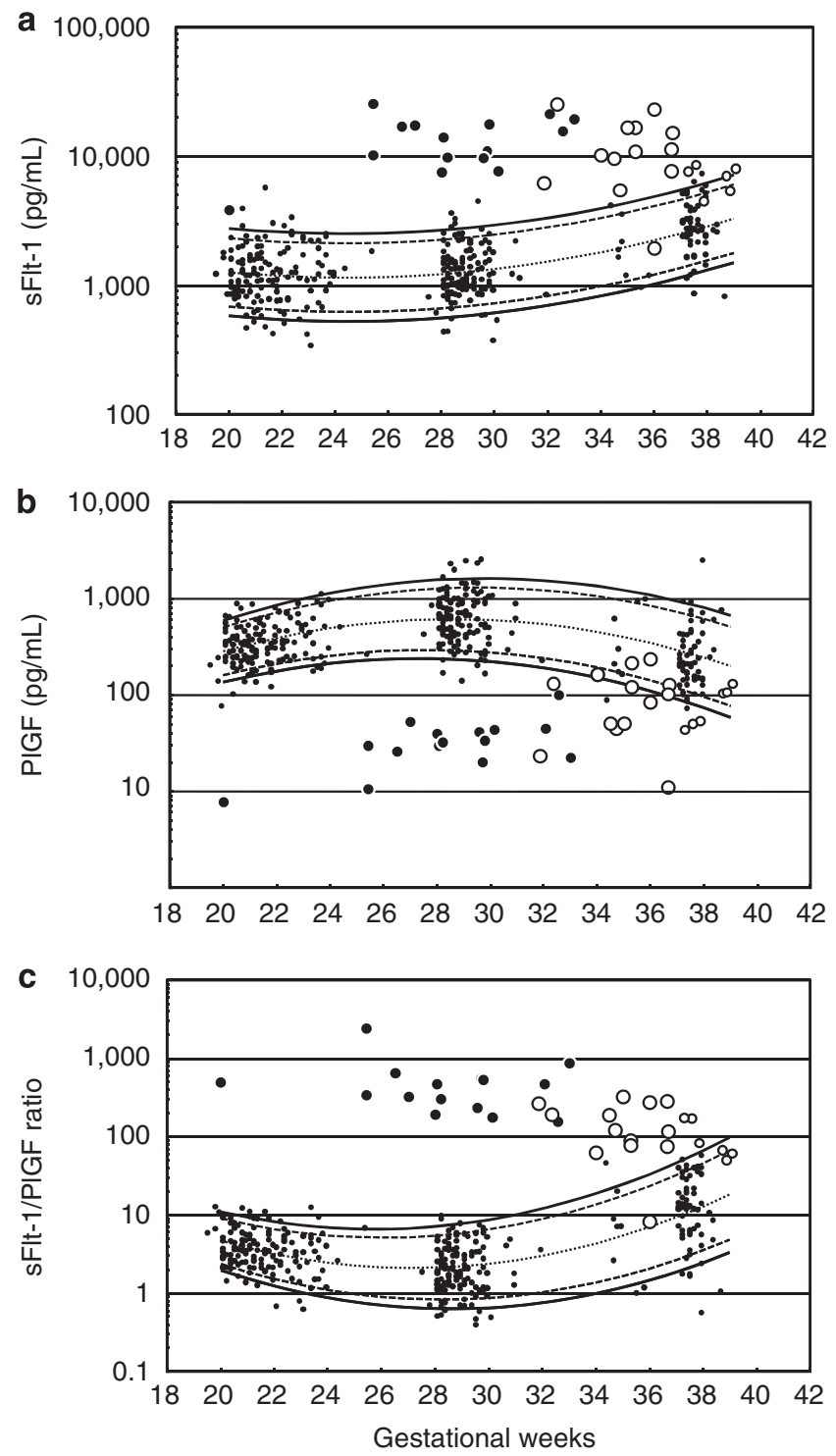

Figure 1 Quadratic curves representing the mean and 5th, 10th, 90th and 95th percentiles of the plasma levels of sFIt-1 (a) and PIGF (b) and the sFIt-1/PIGF ratio (c) from 20 to 38 weeks of gestation. Solid curves represent the 5th and 95th percentiles of the reference values, dashed curves represent the 10th and 90th percentiles, and the dotted curve represents the mean. Small closed circles represent the values in the 144 normal controls. Large closed circles represent the values in the 15 women with early-onset (EO) preeclampsia (PE); large open circles represent the values in the 13 women with late-onset (LO) PE complicated with preterm delivery and medium-sized open circles represent the values in the 6 women with LO-PE with term delivery.

be associated with clinical symptoms such as hypertension or proteinuria in most women with PE, especially those with EO-PE.

To compare the diagnostic power of the s.d.s. of the three indices (sFlt-1, PlGF and the sFlt-1/PlGF ratio) for the diagnosis of all PE cases, we constructed receiver-operating characteristic (ROC) curves using data from 144 normal controls during the second half of pregnancy and 34 women with PE (Figure $2 \mathrm{a}-\mathrm{c}$ ). The area under the curves (AUCs) of sFlt-1, PlGF and the sFlt-1/PlGF ratio were $0.968,0.951$ and 0.975 , respectively. Thus, the AUC of the s.d.s. of the sFlt-1/PlGF ratio was the best among the three indices. Sensitivities for the diagnosis of all PE at the cutoff value of the 95th percentile of sFlt-
1, PlGF and the sFlt-1/PlGF ratio were 91,76 and $88 \%$, respectively (Table 3).

To compare the diagnostic power of the s.d.s. of the sFlt-1/PlGF ratio for the diagnosis of the three classes of PE (all PE, EO-PE and LO-PE), we constructed ROC curves (data not shown). The AUCs of the sFlt-1/PlGF ratio for the diagnosis of all PE, EO-PE and LO-PE were $0.975,1.000$ and 0.955 , respectively. Thus, the AUCs of the s.d.s. of the sFlt-1/PlGF ratio for the diagnosis of EO-PE were larger compared to those for the diagnosis of all PE or LO-PE. Sensitivities for the diagnosis of all PE, EO-PE and LO-PE at the cutoff value of the 95 th percentile of the sFlt-1/PlGF ratio were 88,100 and $79 \%$, respectively (Table 3 ).

To compare the diagnostic power of the raw values of the three indices for the diagnosis of all PE, we constructed ROC curves using data from 68 normal control patients during 34-38 weeks of gestation and 34 women with PE (Figure $2 \mathrm{~d}-\mathrm{f}$ ). The AUCs of sFlt-1, PlGF and the sFlt-1/PlGF ratio were $0.960,0.936$ and 0.981 , respectively. Thus, the AUCs of the raw values of the sFlt-1/PlGF ratio were the best among the three indices. The sensitivities at the cutoff value of the $5 \%$ false-positive rate of sFlt-1, PlGF and the sFlt-1/PlGF ratio were 85, 67 and $97 \%$, respectively (Table 3 ). The respective cutoff values at a $5 \%$ false-positive rate of sFlt-1, PlGF and the sFlt-1/PlGF ratio were $5750 \mathrm{pg} \mathrm{ml}^{-1}, 91 \mathrm{pg} \mathrm{ml}^{-1}$ and 45.3.

ROC curves were constructed to compare the diagnostic power of raw values of the sFlt-1/PIGF ratio for the diagnosis of the three classes of PE (data not shown). The AUCs of the sFlt-1/PlGF ratio for the diagnosis of all PE, EO-PE and LO-PE were 0.981, 1.000 and 0.966, respectively. Thus, the AUC of the raw value of the sFlt-1/PlGF ratio for the diagnosis of EO-PE was large compared to the diagnosis of all $\mathrm{PE}$ or LO-PE. Sensitivities at the cutoff value of a $5 \%$ false-positive rate of the sFlt-1/PlGF ratio for the diagnosis of all $\mathrm{PE}, \mathrm{EO}-\mathrm{PE}$ and LO-PE were 97, 100 and 95\%, respectively. The cutoff values at the 5\% false-positive rate of the sFlt-1/PlGF ratio for the diagnoses of all PE, EO-PE and LO-PE were all 45.3.

\section{DISCUSSION}

We found two novel findings in this study: First, the most appropriate reference range curves for plasma levels of sFlt-1, PlGF and the sFlt-1/ PlGF ratio can be modeled as quadratic curves after logarithmic transformation. Second, the sFlt-1/PlGF ratio showed the best diagnostic power for all PE, EO-PE and LO-PE. In addition, a cutoff value of 45 for the sFlt-1/PlGF ratio resulted in the best sensitivity and specificity for the diagnosis of all PE and EO-PE.

Plasma sFlt-1 and PIGF levels are as reliable as serum sFlt-1 and PlGF levels when using Elecsys sFlt-1 and Elecsys PlGF for the following three reasons. First, we could establish reference ranges for plasma levels of sFlt- 1 and PlGF, and the sFlt-1/PlGF ratio. Second, the distributions of these indices in women with PE in this study were almost the same as in our previous studies using serum samples. ${ }^{1,2}$ In the previous studies, we used ELISA kits from R\&D Systems, which have been used by many researchers for studies of the pathological function of sFlt- 1 and PlGF in women with PE., 5 Third, the correlation determinations between serum and plasma sFlt-1 levels and between serum and plasma PlGF levels were sufficiently high.

Plasma sFlt-1 and PlGF levels determined by Elecsys from Roche Diagnostics were as reliable as plasma sFlt-1 and PlGF levels determined by an ELISA kit from R\&D Systems. The correlation between the ELISA kits from R\&D Systems and Elecsys from Roche Diagnostics for plasma sFlt-1 and PlGF levels and the sFlt-1/PlGF ratio were sufficiently large (all $r>0.9$ using all samples from women with and without PE). For serum sFlt-1 and PlGF levels, the Elecsys assays are 
Table 1 Maternal and infantile characteristics in women with early- and late-onset preeclampsia

\begin{tabular}{|c|c|c|c|c|}
\hline & \multirow[b]{2}{*}{ All women with preeclampsia $(\mathrm{n}=34)$} & \multicolumn{2}{|c|}{ Onset of preeclampsia } & \multirow[b]{2}{*}{ P-value } \\
\hline & & Early onset $(n=15)$ & Late onset $(n=19)$ & \\
\hline Age (years) & $31.7 \pm 5.8$ & $32.4 \pm 5.7$ & $31.2 \pm 6.1$ & 0.543 \\
\hline Prepregnancy body mass index $\left(\mathrm{kg} \mathrm{m}^{-2}\right)$ & $22.7 \pm 4.0$ & $22.4 \pm 4.0$ & $23.0 \pm 4.2$ & 0.633 \\
\hline Nulliparous & $21(62 \%)$ & $10(67 \%)$ & $11(58 \%)$ & 0.728 \\
\hline Gestational age at onset of preeclampsia (weeks) & $32.0 \pm 4.7$ & $27.7 \pm 3.2$ & $35.5 \pm 2.0$ & $<0.001$ \\
\hline Gestational age at delivery (weeks) & $33.4 \pm 4.4$ & $29.5 \pm 3.6$ & $36.5 \pm 1.9$ & $<0.001$ \\
\hline Severe preeclampsia & $28(82 \%)$ & $14(93 \%)$ & $14(74 \%)$ & 0.196 \\
\hline HELLP syndrome & $2(6 \%)$ & $0(0 \%)$ & $2(11 \%)$ & 0.492 \\
\hline Maximum SBP (mm Hg) & $171 \pm 14$ & $177 \pm 13$ & $166 \pm 12$ & 0.017 \\
\hline Maximum DBP (mm Hg) & $106 \pm 11$ & $108 \pm 11$ & $104 \pm 12$ & 0.323 \\
\hline Urinary protein $>2 \mathrm{~g}$ per day & $19(56 \%)$ & $10(67 \%)$ & $9(47 \%)$ & 0.314 \\
\hline Infant birth weight (g) & $1769 \pm 919$ & $977 \pm 378$ & $2395 \pm 708$ & $<0.001$ \\
\hline Small-for-gestational-age infants & $17(50 \%)$ & $11(73 \%)$ & $6(32 \%)$ & 0.037 \\
\hline
\end{tabular}

Abbreviations: DBP, diastolic blood pressure; HELLP, hemolysis, elevated liver enzyme and low platelet; SBP, systolic blood pressure.

Table 2 Plasma levels of sFIt-1, PIGF and the sFIt-1/PIGF ratio in women with early- and late-onset preeclampsia

\begin{tabular}{|c|c|c|c|c|}
\hline & \multirow[b]{2}{*}{ All women with preeclampsia $(n=34)$} & \multicolumn{2}{|c|}{ Onset of preeclampsia } & \multirow[b]{2}{*}{ P-value } \\
\hline & & Early onset $(\mathrm{n}=15)$ & Late onset $(\mathrm{n}=19)$ & \\
\hline Gestational age at measurement (weeks) & $32.6 \pm 4.7$ & $28.4 \pm 3.3$ & $36.0 \pm 2.0$ & $<0.001$ \\
\hline $\log _{10}$ sFlt-1 (pg ml-1) & $4.02 \pm 0.25$ & $4.10 \pm 0.22$ & $3.95 \pm 0.27$ & 0.099 \\
\hline $\log _{10}$ sFlt-1 s.d.s. & $3.71 \pm 1.60$ & $4.75 \pm 1.02$ & $2.89 \pm 1.50$ & $<0.001$ \\
\hline $\log _{10} \mathrm{PIGF}\left(\mathrm{pg} \mathrm{ml} \mathrm{m}^{-1}\right)$ & $1.72 \pm 0.35$ & $1.48 \pm 0.27$ & $1.92 \pm 0.28$ & $<0.001$ \\
\hline $\log _{10}$ PIGF s.d.s. & $-3.38 \pm 1.97$ & $-5.11 \pm 1.32$ & $-2.02 \pm 1.14$ & $<0.001$ \\
\hline $\log _{10}(\mathrm{sF} I \mathrm{t}-1 / \mathrm{PIGF})$ & $2.29 \pm 0.44$ & $2.62 \pm 0.30$ & $2.04 \pm 0.37$ & $<0.001$ \\
\hline $\log _{10}(\mathrm{sFIt-1/PIGF})$ s.d.s. & $4.70 \pm 2.49$ & $6.99 \pm 1.47$ & $2.88 \pm 1.35$ & $<0.001$ \\
\hline
\end{tabular}

Abbreviations: PIGF, placental growth factor; s.d.s., standard deviation score; sFlt-1, soluble fms-like tyrosine kinase 1.

calibrated with recombinant human sFlt-1 and PlGF and standardized against the Quantikine sFlt-1 and PlGF ELISAs (R\&D Systems). ${ }^{7}$ Our study results suggested that Elecsys sFlt-1 and Elecsys PlGF could replace ELISAs (R\&D Systems) as the standard method for measuring plasma sFlt-1 and PlGF levels.

In this study, we found that an arbitrary cutoff value of the sFlt-1/PlGF ratio might be superior to the cutoff values of reference range curves. As the arbitrary cutoff value of 45 for the plasma sFlt-1/PlGF ratio showed the best sensitivity and specificity for the diagnosis of $\mathrm{PE}$, we should use this fixed value instead of reference range values for the diagnosis of PE. In a recently published study, a serum sFlt-1/PIGF ratio cutoff value of 85 had the best sensitivity and specificity of 82 and $95 \%$, respectively. ${ }^{7}$ Because a serum sFlt-1/PlGF ratio of 85 is almost the 95th percentile at 34-36 weeks and because a plasma sFlt-1/PlGF ratio of 45 is almost the 96th percentile at 36 weeks of gestation, the best cutoff values of the sFlt1/PIGF ratio were set at around the 95th percentile at 34-36 weeks of gestation in both studies. Therefore, the best cutoff values for the sFlt-1/ PlGF ratio may be the same for both plasma and serum samples.

Although we claim that the sFlt-1/PlGF ratio provides the best diagnostic accuracy for PE, the result may be different if the ratio is used earlier in pregnancy as a predictor of later PE. In our previous studies, the values of sFlt-1 and the sFlt-1/PlGF ratio in women who subsequently developed $\mathrm{PE}$ gradually deviated from the normal range to the upper area (over the $95 \%$ reference range) of the curve before the onset of PE. ${ }^{3,4}$ Because the upper (95\%) limit of the sFlt-1 value and sFlt-1/PlGF ratio changes markedly according to gestational weeks, ${ }^{3,4}$ the use of reference range curves may be better compared to the use of an arbitrary cutoff value in the prediction of subclinical PE that will soon develop to PE.

In this study, no samples of pregnant women without $\mathrm{PE}$ who developed PE later in pregnancy were included. Therefore, the main value of this study is the reference range for plasma Elecsys sFlt- 1 and Elecsys PlGF in the second half of pregnancy, as well as the values in women with overt PE. We are now examining prospectively collected data for evaluating the possibility of PE prediction using plasma Elecsys sFlt-1 and Elecsys PlGF, although we have not completed the analysis of this prospectively collected data.

Although we constructed normal reference values of plasma Elecsys sFlt-1, Elecsys PlGF and the sFlt-1/PlGF ratio in the second half of pregnancy, sFlt-1 has been recently shown to be elevated as early as at the end of the first trimester. ${ }^{8}$ In another report evaluating serum Elecsys sFlt-1 and Elecsys PlGF and the sFlt-1/PlGF ratio, reference ranges for these values were shown from 10 weeks of gestation until term. ${ }^{7}$ If clinicians use serum samples, they can evaluate the possible functions of Elecsys sFlt-1 and Elecsys PlGF in the prediction of PE.

The definition of PE includes only blood pressure and proteinuria, not angiogenic factors. The measurement of these factors by any method might be important not only in the pathophysiology of PE but also in the prediction of PE onset. ${ }^{1,2,5,6,8}$ Moreover, several studies have already reported the utility of sFlt-1 measurements using ELISA kits for PE diagnosis. ${ }^{9,10}$ However, to our knowledge, the clinical utilities of the sFlt-1/PlGF ratio for diagnosing PE have been evaluated only by our group and another group. ${ }^{7}$ The sensitivities at the cutoff value of 45 of the sFlt-1/PlGF ratio for the diagnosis of all PE, EO-PE 

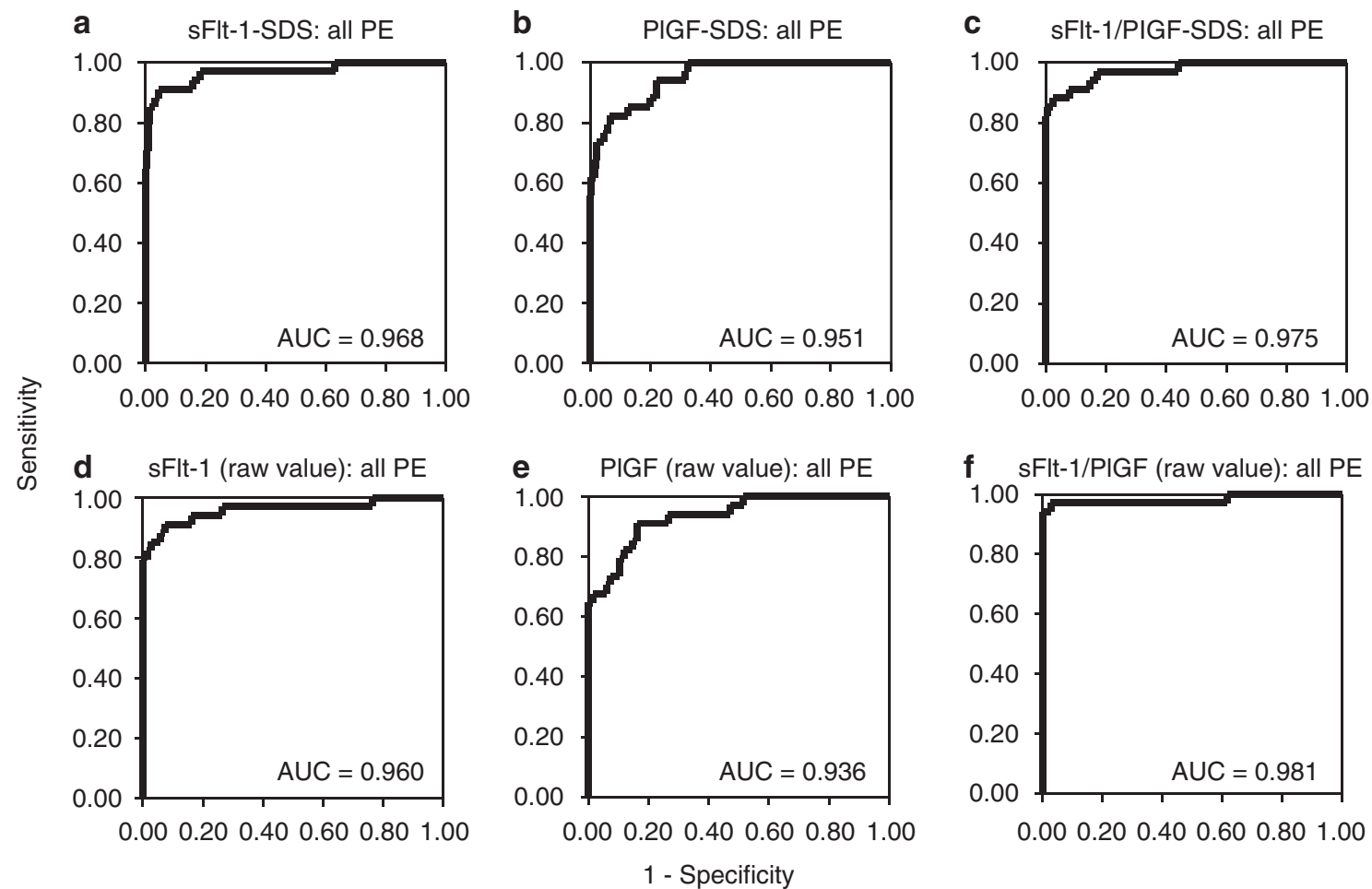

f sFlt-1/PIGF (raw value): all PE

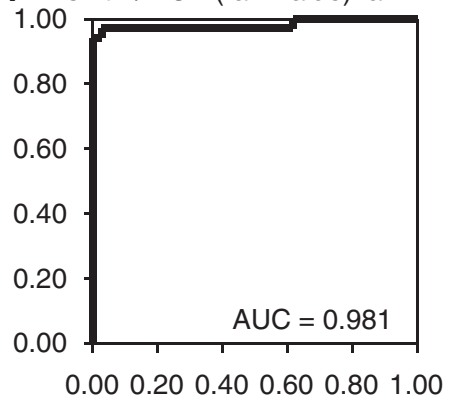

Figure 2 Receiver-operating characteristic (ROC) curves of standard deviation scores (s.d.s.) of sFIt-1 (a), PIGF (b) and sFIt-1/PIGF ratio (c) for the diagnosis of all PE were constructed using 144 normal controls during the second trimester and 34 women with PE. The area under the curves (AUCs) of sFIt-1, PIGF and sFIt-1/PIGF ratio were $0.968,0.951$ and 0.975 , respectively. Thus, the AUC of sFlt-1/PIGF ratio was the best among the three indices. ROC curves of raw values of sFIt-1 (d), PIGF (e) and sFIt-1/PIGF ratio (f) for the diagnosis of all PE were also constructed using 68 normal controls during 34-38 weeks of gestation and 34 women with PE. The AUCs of sFIt-1, PIGF and sFIt-1/PIGF ratio were $0.960,0.936$ and 0.981 , respectively. Thus, the AUC of the sFIt-1/ PIGF ratio was the best among the three indices.

Table 3 AUC and clinical sensitivity at various cutoff values of plasma sFIt-1, PIGF and sFIt-1/PIGF ratio for the diagnosis of PE, EO-PE and LO-PE

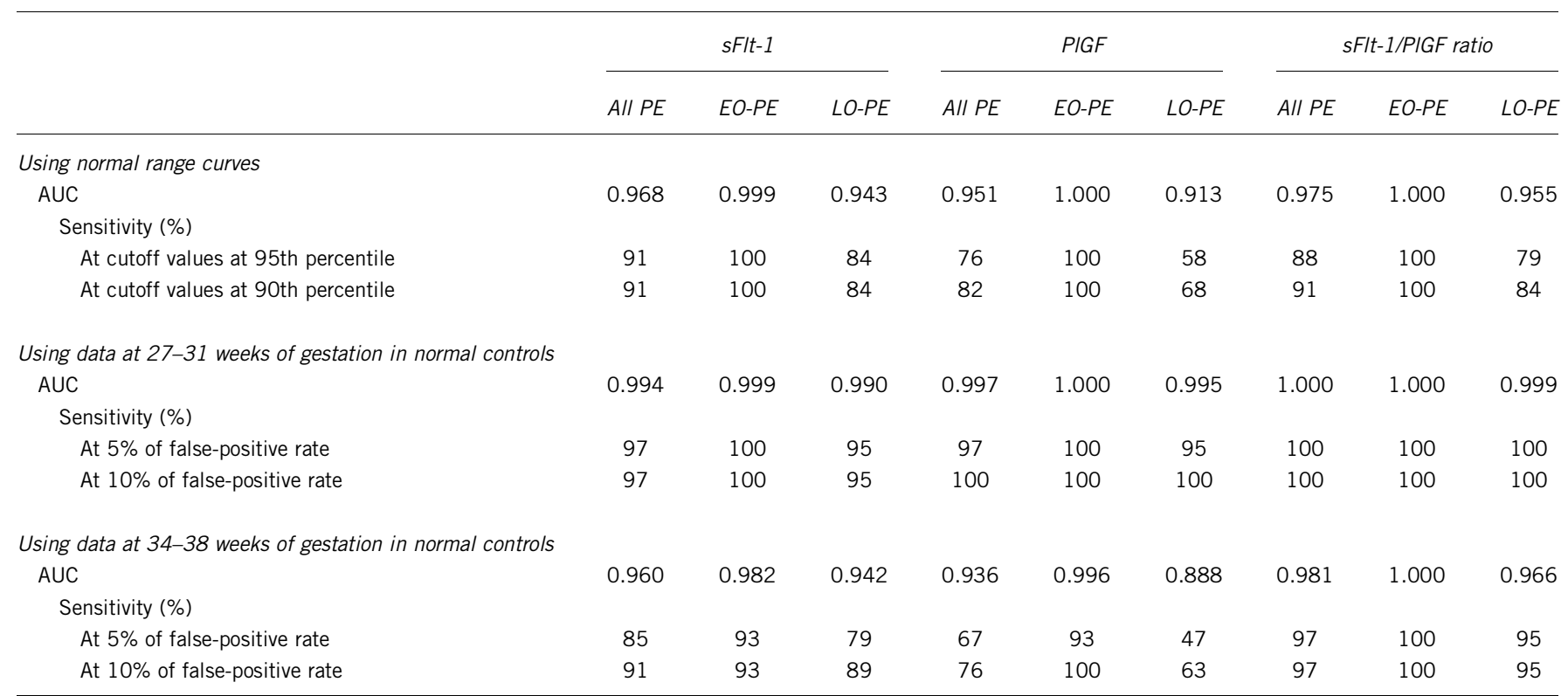

Abbreviations: AUC, area under the curve; EO, early onset; LO, late onset; PE, preeclampsia; PIGF, placental growth factor; sFIt-1, soluble fms-like tyrosine kianse 1.

EO means that the clinical manifestation of preeclampsia occurred at less than 32 weeks of gestation. LO means that the clinical manifestation of preeclampsia occurred at equal to or more than 32 weeks of gestation.

and LO-PE were sufficiently high (97, 100 and 95\%, respectively), with false-positive rates of $5 \%$. In addition, our method requires only 18 min for measuring the sFlt-1/PIGF ratio. ${ }^{7}$ Therefore, we think that the measurement of the sFlt-1/PlGF ratio might assist the diagnosis of $\mathrm{PE}$ and might be helpful for clinicians to judge whether pregnant women with overt PE should be transferred to tertiary centers. 
In conclusion, it appears that measurement of the plasma sFlt-1/ PlGF ratio using Elecsys sFlt- 1 and Elecsys PlGF, using a cutoff value of 45 , might assist in the diagnosis of PE. As the clinicians have long required a rapid and fully automated method to measure plasma/ serum levels of sFlt-1 and PlGF, introduction of Elecsys sFlt-1 and Elecsys PIGF into clinical diagnosis will contribute to the early detection of PE.

\section{CONFLICT OF INTEREST}

The authors declare no conflict of interest.

\section{ACKNOWLEDGEMENTS}

This work was supported by research Grants (18591809) from the Ministry of Education, Science and Culture of Japan. We acknowledge the kind gifts of the Elecsys sFlt- 1 and Elecsys PlGF, and cooperation with the measurements of sFlt1 and PlGF from Roche Diagnostics. We are indebted to Maiko Yuzawa for daily collection of blood samples and for centrifuging and storing samples.

1 Hirashima C, Ohkuchi A, Arai F, Takahashi K, Suzuki H, Watanabe T, Kario K, Matsubara S, Suzuki M. Establishing reference values for both total soluble Fms-like tyrosine kinase 1 and free placental growth factor in pregnant women. Hypertens Res 2005; 28: 727-732.
2 Ohkuchi A, Hirashima C, Matsubara S, Suzuki H, Takahashi K, Arai F, Watanabe T, Kario K, Suzuki M. Alterations in placental growth factor levels before and after the onset of preeclampsia are more pronounced in women with early onset severe preeclampsia. Hypertens Res 2007; 30: 151-159.

3 Sato K. A proposal for a new definition and classification of 'Pregnancy induced Hypertension (PIH)' (2004). In Japan Society for the Study of Toxemia of Pregnancy (ed), Historical Perspective of Study of Pregnancy-Induced Hypertension in Japan. Medical View: Tokyo, 2005, pp 54-87.

4 Ogawa Y, Iwamura T, Kuriya N, Kuriya N, Nishida H, Takeuchi H, Yakada M, Itabashi K, Imura S, Isobe K. Birth size standards by gestational age for Japanese neonates. Acta Neonatologica Japonica 1998; 34: 624-632.

5 Levine RJ, Maynard SE, Qian C, Lim KH, England LJ, Yu KF, Schisterman EF, Thadhani R, Sachs BP, Epstein FH, Sibai BM, Sukhatme VP, Karumanchi SA. Circulating angiogenic factors and the risk of preeclampsia. N Engl J Med 2004; 350: 672-683.

6 Levine RJ, Lam C, Qian C, Yu KF, Maynard SE, Sachs BP, Sibai BM, Epstein FH, Romero R, Thadhani R, Karumanchi SA, CPEP Study Group. Soluble endoglin and other circulating antiangiogenic factors in preeclampsia. N Engl J Med 2006; 355: 992-1005.

7 Verlohren S, Galindo A, Schlembach D, Zeisler H, Herraiz I, Moertl MG, Pape J, Dudenhausen JW, Denk B, Stepan H. An automated method for the determination of the sFIt-1/PIGF ratio in the assessment of preeclampsia [Epub ahead of print Oct 20, 2009]. Am J Obstet Gynecol 2009; 202: 161.e1-161.e11.

8 Baumann MU, Bersinger NA, Mohaupt MG, Raio L, Gerber S, Surbek DV. Firsttrimester serum levels of soluble endoglin and soluble fms-like tyrosine kinase-1 as first-trimester markers for late-onset preeclampsia. Am J Obstet Gynecol 2008; 199: 266.e1-266.e6.

9 Woolcock J, Hennessy A, Xu B, Thornton C, Tooher J, Makris A, Ogle R. Soluble Flt-1 as a diagnostic marker of pre-eclampsia. Aust NZJ Obstet Gynaecol 2008; 48: 64-70.

10 Salahuddin S, Lee Y, Vadnais M, Sachs BP, Karumanchi SA, Lim KH. Diagnostic utility of soluble fms-like tyrosine kinase 1 and soluble endoglin in hypertensive diseases of pregnancy. Am J Obstet Gynecol 2007; 197: 28.e1-28.e6. 